http://dx.doi.org/10.35381/racji.v6i1.1469

\title{
El derecho a la eutanasia en el Ecuador
}

The right to euthanasia in Ecuador

\author{
Karla Alexandra Once-González \\ kaonceg70@est.ucacue.edu.ec \\ Universidad Católica de Cuenca, Cuenca \\ Ecuador \\ https://orcid.org/0000-0003-4227-6315 \\ María Auxiliadora Santacruz-Vélez \\ maria.santacruz@ucacue.edu.ec \\ Universidad Católica de Cuenca, Cuenca \\ Ecuador \\ https://orcid.org/0000-0001-9617-7289 \\ Karenth Marcela Galvis-Martínez \\ karenth.galvis@ucacue.edu.ec \\ Universidad Católica de Cuenca, Cuenca \\ Ecuador \\ https://orcid.org/0000-0003-3816-3799 \\ María Paola Aguilar-Rodas \\ maria.aguilar@ucacue.edu.ec \\ Universidad Católica de Cuenca, Cuenca \\ Ecuador \\ https://orcid.org/0000-0003-2689-392X
}

Recepción: 15 de septiembre 2021

Revisado: 25 octubre 2021

Aprobación: 15 de noviembre 2021

Publicación: 01 de diciembre 2021 


\title{
RESUMEN
}

En este artículo se realiza un análisis acerca de la eutanasia, como un derecho fundamental que el Estado ecuatoriano debería proporcionar a sus residentes, para proteger los principios de la autonomía y autodeterminación, así como un modelo bioético basado en la deliberación por parte del paciente, pues a la fecha, a nivel interno no se garantiza el mismo, lo cual es contrario a la facultad personal del goce de una muerte en condiciones de dignidad.

Descriptores: Ética médica; eutanasia; derecho a la vida. (Tomado del tesauro de la UNESCO).

\begin{abstract}
This article makes an analysis about euthanasia, as a fundamental right that the Ecuadorian State should provide its residents, to protect the principles of autonomy and self-determination, as well as a bioethical model based on deliberation by the patient, as to date, internally, it is not guaranteed, which is contrary to the personal faculty of the enjoyment of a death in dignity conditions.
\end{abstract}

Descriptors: Medical ethics; euthanasia; right to life. (Taken from the UNESCO thesaurus). 


\section{INTRODUCCIÓN}

La vida es considerada como un bien superior, en muchas culturas la vida es celebrada y la muerte es una ocasión de tristeza; en otras culturas la vida es llegar a un mundo a conocer la tristeza mientras que la muerte es una fiesta; por lo tanto, la muerte y la vida son fenómenos biológicos que han acompañado a la humanidad durante su desarrollo. El concepto de eutanasia ha sido manejado por 2 tipos de hombres, los hombres de ciencia y los religiosos; los cuales se basan en sus conocimientos o creencias, desarrollando así la dignidad humana, tanto para defenderla o rechazarla (Creagh, 2012). Con base en estos conceptos, existen quienes se encuentran a favor de la eutanasia 0 dignidad de los enfermos a tener el derecho a una muerte digna, y quienes se encuentran en contra de ésta, bien sea por creencias religiosas o por objeción de conciencia. Al margen de la creencia que se pueda tener en tal sentido, "el hombre debe mirar a su alrededor, escuchar y preguntar el porqué de una decisión como esta, buscar la mejor manera de aliviar al ser humano de sufrimientos" (Creagh, 2012).

Es claro que, en el Ecuador, no existe una política de estudio y reconocimiento acerca de la eutanasia como una forma de muerte digna, a pesar de pertenecer al Sistema Interamericano de Derechos Humanos, siendo un tema que no ha sido tratado adecuadamente, pues si bien se ha hablado de políticas acerca de cuidados paliativos, y se han elaborado protocolos para su tratamiento, en la actualidad no se ha aceptado el ejercicio de la misma como un derecho fundamental. La eutanasia es un derecho fundamental para las personas quienes se encuentran en tratamientos de cuidados paliativos, pero sus opciones han terminado y presentan cuadros de mucho dolor que le impiden tener una vida digna.

Sin embargo, y pese a lo anterior, las políticas de Estado no permiten su práctica que dista mucho de un suicidio o suicidio asistido, siendo un tema completamente controversial, el cual por supuesto genera personas a favor y en contra de la misma. El Ecuador cuenta con un contenido obligacional claro, en cual indica que todas las personas deben gozar de los mismos derechos sin discriminaciones, pero al hablar de 
un tema tan delicado como es la eutanasia, a nivel estatal no hay una respuesta contundente que garantice los derechos de estas personas, según esos deberes constitucionales.

Esto, porque a nivel constitucional, se toma en cuenta la vida digna de las personas, pero no se incluye en ese nivel de protección a los enfermos terminales quienes no tienen una vida en condiciones de dignidad al encontrarse en el final de su vida, con una patología terminal que le genera una condición precaria de existencia. Por esto, una de las finalidades de este artículo, es hacer una comparación con otros sistemas jurídicos, los cuales han entrado a este tema que están luchando por políticas para el respeto de los derechos de estas personas.

\section{Referencial Teórico}

\section{La eutanasia}

En el Ecuador, la eutanasia no cuenta con un estudio académico a profundidad, a pesar de que otros países como Colombia y España ya la han legalizado, no ha terminado de ser un tema de controversia. Al revisar la bibliografía, se tiene un primer trabajo en que el autor indica varios artículos de la legislación de algunos países en los cuales se indica a la eutanasia como un derecho fundamental de los pacientes con diagnóstico de una enfermedad terminal, por lo tanto, en otros países han iniciado la legislación sobre una muerte digna en aquellos pacientes que ya han llegado al final de su vida (Guairacaja, 2011).

Mientras tanto en Ecuador en la legislación aún se sigue manteniendo que la vida es el principal derecho del ser humano, pero también hay que recalcar que el Ecuador pertenece a organismos internacionales y por lo tanto, deben igualmente respetar los conceptos que estos presentan, y al estar dentro de un sistema internacional también se debe adoptar las leyes y reglamentos que estos defienden, entonces podríamos decir que nuestros legisladores a pesar de conocer de qué estamos dentro de un sistema no 
han planteado el respeto y el derecho a la muerte digna de los pacientes terminales, siendo así la eutanasia un tema no abordado en la asamblea nacional.

En el mencionado trabajo, el autor realizó una encuesta para obtener opiniones acerca de este tema controversial, realizándola tanto a profesionales de la salud como a los de derecho, determinando así que aún hay limitaciones dentro de los pensamientos jurídicos y médicos, entre estos muchos concuerdan en la legalización de la eutanasia, pero al mismo tiempo en el personal de salud se encuentra la interrogante de que el juramento que se realiza impide que esta práctica sea realizada. Igualmente, se tiene otro trabajo de investigación realizado en Septiembre 2014, en que el autor también busca dar un punto de vista sobre la eutanasia como un derecho fundamental, que debería ser estudiado por el Estado ecuatoriano, y analiza algunos casos que se han dado en otros países, recabando información de todos los artículos que pueden defender la teoría del derecho a su práctica (Baños, 2014).

\section{La bioética}

La bioética es una rama que estudia los principios por los cuales indica la actuación que se tiene sobre asuntos de la vida; es decir que es lo que se debe hacer y no se debe hacer. La bioética norma los actos médicos donde la tecnología y estudios científicos avanzan rápidamente (Millás-Mur, 2019). Uno de los aspectos esenciales que se deben tener en cuenta, es que un asunto tan complejo como lo es la eutanasia, sólo se puede comprender desde un análisis bioético, pues su dimensión escapa, tanto de lo jurídico, como de lo médico, si se tiene en cuenta el hecho de que, ni son los abogados, ni son los médicos, quienes deben tomar decisiones sobre un tema tan trascendental, sino que es la persona misma quien se encuentra en tal condición, quien debiera ser la única titular de este derecho fundamental a tomar la decisión sobre la forma en que considera que debe terminar su vida. 


\section{MÉTODO}

El presente trabajo investigativo es de carácter descriptivo, jurídico y documental. Por consiguiente, la investigación y conocimiento científico en lo relacionado a lo jurídico, debe realizarse teniendo en cuenta la hermenéutica jurídica, toda vez que sirve a la enseñanza y aprendizaje del derecho, dando guía para tener un fuerte conocimiento sobre el campo jurídico, por lo cual es fundamental que contenga una crítica que sea capaz de aportar al nuevo conocimiento (Matías-Camargo, 2012).

Por lo mencionado con anterioridad y tal como se evidenciará con el análisis de documentos, a partir de los cuales se pretende destacar una investigación que abra paso al nuevo conocimiento en lo que atañe a la eutanasia en el Ecuador, a fin de alcanzar los objetivos que fueron propuestos. Se trata de una metodología descriptiva, en el sentido de que presenta la realidad de la manera en la que se percibe en una situación de espacio-tiempo, es decir, se hace una descripción del fenómeno. En el mismo sentido, se trata de una investigación teórica, toda vez que se da el análisis, la síntesis, la crítica y la reelaboración conceptual, del mismo modo, se permite la reconstrucción y la exploración de conceptos para la generación de conocimiento académico. (BarahonaQuesada, 2013).

Del mismo modo, la técnica de análisis documental establece que es un conjunto de operaciones intelectuales por medio de los cuales se busca describir y presentar documentos en forma sistematizada para facilitar su recuperación, en ese sentido, también comprende el procesamiento analítico-sintético (Dulzaides-Iglesias \& MolinaGómez, 2004). De este modo, utilizando la referida técnica, se pudo presentar el contenido de este trabajo investigación, haciendo énfasis en las fuentes bibliográficas en lo relacionado a la eutanasia en el Ecuador y en el sistema comparado.

De la manera en la que se evidencia en el trabajo, se realizó un análisis jurídico-crítico a partir de la doctrina sobre la materia. En el mismo sentido, se tuvieron en cuenta postulados internacionales que han sido emitidos en lo relacionado solo al sector de la salud, también se utilizaron diferentes referentes bibliográficos, como lo son artículos de 
investigación, tesis de grado, libros en formato electrónico y físico, los cuales basan su objeto de estudio.

\section{RESULTADOS}

Se presenta el análisis sintético - teórico de los resultados de la investigación:

\section{Enfermedades terminales, catastróficas, huérfanas, físicas y psíquicas}

Para comprender el alcance complejo de una práctica como la eutanasia, es determinante caracterizar qué son las enfermedades terminales, huérfanas, físicas y síquicas, para efectos de establecer, hasta qué punto su presencia afecta de manera directa las condiciones de vida digna de una persona. El primer concepto al que debe hacerse alusión, es al de enfermedad terminal, la cual puede entenderse como aquella "avanzada e irreversible con síntomas múltiples, impacto emocional, pérdida de autonomía, con muy escasa o nula capacidad de respuesta al tratamiento específico y con un pronóstico de vida limitado a semanas o meses" (Navarro-Sanz, s.f. p.1). Este concepto inicial tiene una característica, y es que, a pesar de que hace mención a un factor de inmediatez en cuanto al acaecimiento de la muerte, no fija como tal cuál es el período de tiempo que debe establecerse como fecha límite para ello.

Por esta razón, resulta interesante traer a colación el concepto que se ha establecido en un sistema como el colombiano, en el cual sí está permitida la práctica de la eutanasia, entendiendo que se trata de una enfermedad terminal, aquella "que se caracteriza por la ausencia de posibilidades razonables de respuesta al tratamiento, por la generación de sufrimiento físico - psíquico a pesar de haber recibido el mejor tratamiento disponible y cuyo pronóstico de vida es inferior a seis meses" (Resolución 971 de 2021).

En lo que concierne a este trabajo, se considera un error delimitar el concepto de terminalidad de una enfermedad con una fecha en particular, pues existen un sinfín de variables que se pueden presentar en el desenlace de una patología; sin embargo, al margen de establecer un tiempo concreto, lo cierto es que el carácter terminal de una 
enfermedad, sí tiene que ver, en gran medida, con la inminencia de la ocurrencia del evento mortal.

\subsection{Cáncer}

Es la formación de tejidos que crecen de forma desordenada, rápida e irreversible, estas células que crecen son diferentes a las células precursoras, alterando la función normal y van depender exclusivamente del huésped para su crecimiento e irrigación, se debe diferenciar que el cáncer son neoplasias por lo general malignas y su diagnóstico se genera a través de estudios histológicos, y con el paso del tiempo si no se los localiza con tiempo pueden provocar metástasis a diferentes lugares del cuerpo. (GonzálezRodríguez, 2007). En cuanto a los tipos de cáncer más comunes, se podría indicar el cáncer de mama, estómago, tiroides, cervicouterino, osteosarcoma, de próstata, pulmón, colon, leucemias, entre otros. Los tratamientos dependerán de la clase de cáncer, pero por lo general se realiza Exceresis del tumor, seguido de un tratamiento a base de quimioterapia; en las fases finales se indica tratamiento de dolor y cuidados paliativos.

\subsection{Síndrome de Inmunodeficiencia Adquirida.}

Es la fase crítica de la infección por $\mathrm{VIH}$, en esta etapa el sistema inmunológico de la persona esta tan deteriorado que no puede reaccionar a las infecciones y el cuerpo se desgasta poco a poco, por lo que la persona puede desarrollar graves infecciones, una persona que sea diagnóstica en esta fase tiene probabilidades bajas de supervivencia, con una tase de fallecimiento a los 3 años, por lo general fallecen a los 3 años, entre las manifestaciones clínicas se cuenta con neumonías provocadas por sarcoma de Kaposi, Pneumocitis Carinii, encefalopatías, síndrome de desgaste que provoca fiebres elevadas, hepato-esplenomegalia, diarreas. (Chávez \& Castillo, 2013). 


\subsection{Enfermedad Catastrófica o Grave}

Se trata de aquellas patologías que se caracterizan por tener un curso crónico, del cual se colige un riesgo alto, y su tratamiento resulta de alto costo desde el punto de vista económico y por el impacto social, la cual, por tener un carácter extendido en el tiempo o permanente, resulta ser susceptible de programación". (Acuerdo Ministerial 1829, p. 2).

\subsection{Encefalopatía hepática grado III - IV con síndrome hepatorrenal}

Consiste en un trastorno neuro-psiquiátrico que se produce en los pacientes con disfunción hepática grave, el cual tiene como característica, que se trata de una acumulación severa de amonio que es generado por el intestino grueso, y en el cual, quienes la padecen, pueden presentar desde pérdida de conciencia hasta un coma profundo. (Bustíos, 2007) Este síndrome es una de las complicaciones más comunes de los pacientes quienes padecen de hepatopatía grave, el cual es caracterizado por una lesión perenal, que no cede a la expansión de volumen, es de baja prevalencia, pero de mortalidad elevada, debido a que el $50 \%$ de los pacientes fallecen si no se da tratamiento oportuno. (Varela-Jiménez, 2015).

\subsection{Insuficiencia Renal Crónica}

Según Gómez, es una pérdida progresiva, irreversible y permanente de la función renal por filtración glomerular, que consiste en un daño renal persistente producido al menos durante 3 meses, que disminuye la cantidad de nefronas lo que se caracteriza por una disminución en la excreción. Debido a que el riñón pierde la capacidad de eliminación se puede provocar una acumulación de azoados (urea, creatinina); provocando síntomas caracterizados por irritabilidad, letargia, anemia, coagulopatías entre otras; la cual tiene como tratamiento la diálisis o el trasplante renal. (Gómez-Sánchez, 2002). 


\subsection{Demencia muy avanzada}

Consiste en una patología síquica que se caracteriza por producir alteraciones a nivel de la memoria, aprendizaje, concentración, lenguaje, comportamiento; se asocia con conductas que afectan el desarrollo físico, y en consecuencia, el entorno social, familiar y laboral, la cual se puede desarrollar de múltiples formas, siendo el Alzheimer la que presenta mayor prevalencia en la población entre los 65-70 años. Esta patología ocasiona gran dependencia de las personas que las padecen y puede inclusive provocar alteraciones en las comorbilidades presentes. (Formiga et al. 2009).

\subsection{Enfermedades cerebro vasculares}

Muños indica que se trata de un grupo de enfermedades que afectan directamente al sistema nervioso, ya sea por baja irrigación o por déficit de riego de nutrientes, lo que ocasiona el deterioro del tejido cerebral, afectando de diferentes maneras las funciones motoras, sensitivas del cuerpo. Dentro de ellas, se pueden presentar afecciones isquémicas y hemorrágicas, por lo cual sus síntomas pueden ir desde una leve alteración del movimiento, hasta parálisis completas como paraplejías, cuadriplejías etc., siendo enfermedades con una gran incidencia de limitaciones que pueden provocar la aparición de otras enfermedades a nivel de la piel, pulmonar, entre otras. (Muñoz-Collazos, 2010).

\subsection{Enfermedad Huérfana o Rara}

Se trata de aquellas potencialmente mortales o debilitantes a largo plazo, y aunque se caracterizan por ser de baja prevalencia, tienen un importante inconveniente, y es son de alta complejidad, constituyendo un conjunto amplio y variado de trastornos que tienen en común el ser crónicos y altamente discapacitantes. (Resolución 971 de 2021, p.2).

\subsection{Adrenoleucodistrofia ligada al cromosoma $X$.}

Consiste en una enfermedad hereditaria ligada al cromosoma $X$ que es autosómica recesiva, la que implica acumulación de ácidos grasos a nivel de las vainas de mielina, 
provocando daños a nivel neuronal y a nivel de la glándula suprarrenal, siendo producida en la infancia y ocasionando daño a nivel de la materia blanca. Esta enfermedad provoca la muerte en un plazo de 5 a 10 años aproximadamente, y tiene entre sus síntomas específicos la paraparesia espástica, lo que provoca muchas limitaciones en el desarrollo normal de los niños y ocasiona su muerte a futuro por la desmielinización de la sustancia blanca. (Delgado-Rojas, 1999).

\subsection{Enfermedad de Huntington}

Enfermedad conocida como el mal del San Visto, se trata de una enfermedad autosómica dominante, que se produce por una afectación a nivel subtalámico provocando en los pacientes la presencia de alteraciones del comportamiento, alteraciones afectivas, alteraciones motoras y se caracteriza por la presencia de movimientos involuntarios, provoca disartria y en casos avanzados presencia de signos de Babinski. La edad de aparición puede ser entre los 20-30 años comúnmente. También los síntomas de depresión y psicosis son propios del inicio de la enfermedad, provocando que las personas que lo padecen disminuyan en la calidad de vida, por las afectaciones producidas. (Rodríguez et al. 2013).

\subsection{Esclerosis múltiple}

Es una enfermedad desmielinizante, autoinmune e inflamatoria que afecta a todo el sistema nervioso central, ocasionando lesiones incapacitantes en los adultos jóvenes, su sintomatología puede ser remitente, como progresiva hasta causar la muerte. Las manifestaciones clínicas pueden ser trastornos sensitivos como motores, puede cursar con cuadros de neuritis óptico, neuralgia del trigémino, ataxia, vejiga neurogénica, ataxia, disartria, convulsiones, demencia, entre otras es una enfermedad limitante en cuando al desarrollo físico dependiendo la fase en la que se encuentre el paciente. (Domínguez, 2012) 


\subsection{Esclerosis lateral amiotrófica}

También conocida como enfermedad de moto neuronas, se caracteriza por una destrucción axonal de neuronas motoras, que conlleva una disminución gradual del funcionamiento de las neuronas motoras de la corteza cerebral -con su posterior muerte, provocando así una parálisis de todos los músculos, con pronóstico sombrío debido a que con el paso de los años se produce parálisis de los músculos respiratorios lo que ocasiona la muerte. (Bucheli, 2012).

\subsection{Espondilitis anquilosante juvenil}

Es una enfermedad autoinmune y progresiva que afecta a las articulaciones especialmente la sacro ilíacas y columna lumbar-, cuyas manifestaciones clínicas características son dolores y rigidez matutina que mejorara con el ejercicio, pero con el paso del tiempo el dolor se exacerba irradiándose hacia los glúteos, provoca disminución de la movilidad espinal, generando a su vez dolores intensos a la persona que padece esta enfermedad limitando sus actividades diarias. (González-Rodríguez, 2013)

\subsection{Enfermedad Creutzfeldt-Jakob}

Es una enfermedad degenerativa que se presenta con un deterioro rápido y progresivo de la memoria, se acompaña de cambio de comportamiento, alteraciones visuales y falta de coordinación, suele presentar alrededor de los 60-70 años por el deterioro neuronal progresivo que presenta. Esta enfermedad suele ser muy limitante en los pacientes debido a que ocasiones cuadros de demencia con progreso rápido que puede ocasionar la muerte. (Munné-Díaz, 2013).

\subsection{Fibrosis quística con afectación pulmonar}

Es una enfermedad hereditaria que puede ocasionar daño a nivel gastrointestinal, pulmonar $u$ otros órganos del cuerpo. Las alteraciones a nivel pulmonar provocadas por esta patología, son la producción de secreciones espesas y viscosas a nivel de los 
bronquios, lo que ocasiona que los pacientes presenten cuadros de tos que moviliza secreciones verdosas provocando que se colonice bacterias que provocan daño del epitelio pulmonar, y este daño, a su vez ocasiona que el paciente presente necesidades de oxígeno, limitando sus actividades físicas. (Mendoza, 2018).

\subsection{Esquizofrenia}

Es un trastorno psiquiátrico grave, que cursa con una variedad de signos y síntomas como son la presencia de delirios, alucinaciones, pérdida de la atención, concentración, juicio, pensamientos desordenados que llevan al paciente a estar fuera de la realidad y muchas veces provoca que se ocasione daño, o presente ideas suicidas. En esta patología, quien la padece tiende a permanecer sola, llevándola a estados depresivos que pueden llevar a las ideas suicidas. (Martín, 2009).

\subsection{Osteogénesis imperfecta}

Es un trastorno genético que se caracteriza por una alteración en la formación de colágeno, ocasionando que una fragilidad a nivel ósea y provocando un alto índice de facturas, que, dependiendo el grado de la enfermedad, pueden ser facturas leves e inclusive facturas graves que pueden provocar aplastamientos vertebrales como consecuencia de este aplastamiento daño medular. Los pacientes con esta patología presentan una vida limitada por su riesgo de facturas. (Bou-Torrent, 2020).

\subsection{Síndrome de Cotard}

También conocido como delirio de negación, se caracteriza por delirios en cuanto a que la persona se encuentra muerta o partes del cuerpo de una persona están muertos; esta ideas se generan debido a que la persona se encuentra en una etapa de sicosis severa o en depresión, en el cual tiende a hacerse daño para deshacerse de la parte que él considera muerta, provocando eventualmente desde el corte de un miembro hasta el 
suicidio, resultando una patología muy limitante pues la persona se encuentra totalmente fuera de la realidad. (Figueroa, 2016)

\subsection{Angioedema Hereditario tipo I}

Es una enfermedad rara y de origen genético que se transmite de forma autosómica dominante y que se caracteriza por la presencia de angioedema, sin urticaria ni prurito; puede afectar la piel, las vías respiratorias, sistema gastrointestinal, entre otras partes del cuerpo; no obstante, cuando afecta a las vías respiratorias puede ocasionar un edema intenso ocasionando obstrucción que puede provocar la muerte si no se trata a tiempo. (Vives, 2015)

\section{Análisis sobre la afectación de las enfermedades en los pacientes}

Una vez realizado un concepto claro acerca de lo que son las enfermedades terminales, catastróficas y huérfanas, se realizara un análisis de cómo afectan están patologías a las personas que las padecen, pues a partir de allí, será más fácil comprender el por qué la eutanasia podría resultar una garantía de una muerte en condiciones de dignidad. Entre las enfermedades terminales más conocidas, se tienen todos los tipos de cáncer -sobre todo en las fases terminales-, que presentan metástasis a diferentes partes del cuerpo, dependiendo el tipo de cáncer que sea el originario.

En cases terminales, estas personas por lo general tienden a presentar dolores intensos, para el cual se administra medicación que alivia el dolor por períodos cortos pero que también provocan efectos secundarios como náuseas intensas, vómitos, razón por la cual muchas personas indican que no tienen una calidad de vida adecuada. Personas en fases terminales del SIDA, también tienden a tener varias complicaciones, lo que ocasiona que su calidad de vida se vea deteriorada, pues muchas veces la medicación que se utiliza alivia un síntoma, pero provoca otro tipo de síntomas ocasionados por los efectos secundarios propios de la medicación. 
Mientras tanto, una persona que presenta una enfermedad catastrófica, como lo dice su definición, al ser enfermedades crónicas que se desarrollan con el paso de los años, pero cuando llegan a su fase terminal, pueden ocasionar a las personas muchos efectos tanto físicos como psicológicos. Dependiendo de cada enfermedad, pueden ocasionar que una persona se encuentre en una cama con limitación en su movilidad esto provoca muchas veces que la persona que lo padece sienta que es un peso para su familia y debido a las complicaciones que puede llegar a provocar los pacientes pueden presentar infecciones en diferentes sitios de su cuerpo lo que disminuye aún más su calidad de vida.

Tanto enfermedades terminales, catastróficas y enfermedades raras, pueden ocasionar la presencia de dolores intensos, además de la fuerte limitación en las funciones físicas como la habilidad para realizar actividades diarias sin necesitar la ayuda de un tercero. Sin embargo, también existen patologías que, a pesar de no ser terminales, suelen ser gravemente discapacitantes como las enfermedades psiquiátricas, pues la persona que la padece puede muchas veces se encuentran fuera de la realidad y se ocasiona daño provocando una limitación en su vida.

De esta forma, se puede afirmar que existen múltiples patologías que pueden hacer de la vida, una situación cruelmente dolorosa e insufrible, acentuando así la idea de que no necesariamente el estar vivo es el mejor estado del ser humanos, pues atendiendo a cada caso y cada persona, se puede inferir que algunas veces, la calidad de la existencia puede estar tan deteriorada, que resulta inevitable experimentar una merma en la dignidad de quien la padece, e incluso de sus personas más cercanas.

\section{La eutanasia: Regulación en el Ecuador y en algunos países suramericanos}

La eutanasia proviene de 2 vocablos griegos: Eu bueno y Thanos muerte, lo que permite dar una definición de que eutanasia es el buen morir, teniéndose que algunos autores la definen como un acción en la que el médico produce la muerte de un paciente con enfermedad terminal sin dolor siempre y cuando él mismo lo solicite, por lo cual se podría decir que la eutanasia o buena muerte, es aquella en la que una persona con una 
enfermedad terminal o incurable tras una agonía llena de dolor y que es considerada como cruel por la persona que la padece, pide terminar con su vida. Ahora bien, se debe diferenciar igualmente ciertos criterios o palabras que se encuentran dentro de esta investigación, y que no deben ser confundidos, como son la distanasia, la adistanasia y la ortotanasia (Baum, 2017).

La distanasia es un término diferente a la eutanasia, pues etimológicamente significa dificultad para morir, es decir en la Distanasia prolonga la vida o el sufrimiento de un paciente que se encuentre con una afectación grave psicofísica, esta puede ocurrir mediante la utilización de métodos artificiales. (Esquivel, 2003) La adistanasia, que resulta diametralmente opuesta a la distanasia implica no poner obstáculo a la muerte del paciente mediante la utilización de métodos que ayuden a prolongar su vida, respetando el proceso natural del morir. (Esquivel, 2003)

Por su parte, la ortotanasia: es un término en el cual se mantiene la postura de que la muerte ocurra en el momento adecuado, sin adelantarla ni prolongarla con ningún método artificial, pero garantizando al paciente de métodos paliativos para preservar su dignidad. (Esquivel, 2003)

\section{Diferentes clasificaciones de la eutanasia}

La eutanasia puede ser clasificada según el modo, pudiendo ser activa cuando es producida por una acción que provoca la muerte o pasiva cuando se incurre en una omisión en el tratamiento del paciente y esto ocasiona la muerte del mismo; según la intención, pudiendo ser directa cuando el acto ocasiona la muerte de forma deliberada o indirecta por una acción secundaria que provoca la muerte como por ejemplo el uso de analgésicos; según la voluntad del paciente, pudiendo ser voluntaria aquella que se realiza por voluntad propia del paciente o no voluntaria la que se practica sin tener el consentimiento del paciente-. 


\section{La eutanasia en el Ecuador}

La eutanasia es un tema que no está contemplado y hace un tiempo no es una opción a considerar, a pesar de que en otros países ya se considera una opción legítima para los enfermos terminales y con enfermedades catastróficas. En el Ecuador la eutanasia no está legalizada, pues el principal derecho a nivel interno es la vida, por lo cual, la petición del paciente para que se realice una eutanasia, está muy lejos de ser parte del respeto de la autonomía de los pacientes terminales.

Si bien la Constitución se basa en el derecho a una vida digna como el bien superior, también se debe discutir el caso de aquellas personas que perdieron esa vida digna por alguna enfermedad. El numeral 1 del artículo 3 de la Constitución garantiza sin ninguna discriminación "el efectivo goce de los derechos establecidos en la Constitución y en los instrumentos internacionales, en particular la educación, la salud, la alimentación, la seguridad social y el agua para sus habitantes". (Constitución de la República de Ecuador, 2018, Art.3)

No obstante, en el Artículo 66 de la constitución "Se reconoce y garantiza a las personas: 1.- El derecho a la inviolabilidad de la vida. No habrá pena de muerte." (Constitución de la República de Ecuador, 2018, Art. 66), y por ello, este artículo tratar de legalizar o realizar un estudio a la eutanasia que no se puede realizar adecuadamente, pero también la constitución habla de tener una vida digna, pues los pacientes con enfermedades terminales y catastróficas que se encuentran en el final de sus cuidados paliativos no tienen esta vida digna, al contrario, tiene sufrimiento y dolor.

El Ministerio de Salud Pública ecuatoriano expidió un código de ética médica en el cual se dispuso que "El médico no está autorizado para abreviar la vida del enfermo. Su misión fundamental frente a una enfermedad incurable será aliviarla mediante los recursos terapéuticos del caso." (Código de Ética Médica, 1992, Art. 90).

En esta disposición se indica exclusivamente que el médico sólo realizará en los pacientes en fases terminales de enfermedades cuidados paliativos y el acompañamiento 
que necesiten los familiares, en ningún momento permite que se realice una muerte digna a estos.

En el Código Penal se tiene el Artículo 144 que prescribe el delito de homicidio como "La persona que mate a otra será sancionada con pena privativa de libertad de diez a trece años." (Código Integral Penal, 2014, Art. 144), indicando acerca de la sanción por homicidio culposo, pero en la constitución del Ecuador a diferencia de Colombia, no existe un artículo en el que se contemple la asistencia para final de vida de los pacientes con enfermedades terminales.

Basado en esto, se aprecia que la legislación del Ecuador tiene un desarrollo escaso en cuanto al estudio de la muerte digna, pues es un tema abandonado por parte de los legisladores, siendo determinante que el Ecuador considere la posibilidad de establecer una legislación en cuanto a un tema muy delicado y controversial, sin dejar de brindar atención a este grupo prioritario sino para aquellas personas que desean acogerse a este medio lo hagan dignamente.

\section{En Chile}

En Chile se ha planteado la figura del consentimiento, según el cual las personas tienen el derecho de "otorgar o denegar su consentimiento para someterse a cualquier procedimiento invasivo, de cirugía mayor o compleja, y otros que defina el Ministerio de Salud, salvo que la negativa pueda implicar su muerte y siempre que no exista otro procedimiento alternativo". (Zúñiga-Fajuri, 2008, p.8). Ahora bien, bajo esta aclaración del consentimiento, el paciente tiene la autonomía de aceptar o rechazar el tratamiento 0 procedimientos a realizar aun sabiendo que puede ocasionarle la muerte, pero debe cumplir con los requisitos; de que se trate de paciente en un estado terminal producto de su enfermedad grave o incurable, y que los cuidados paliativos brindados sean innecesarios y solo prolongue su vida. (Zúñiga-Fajuri, 2008).

Rodolfo Figueroa en su trabajo se realiza las siguientes preguntas: ¿Está justificado desconectar a un paciente terminal? ¿Está permitido suministrarle una dosis de opiáceos 
destinada a aliviar su sufrimiento, incluso previendo que pueda causar su muerte? ¿Está permitida la eutanasia? ¿Estos se relaciona con derechos de autonomía de los pacientes?, son preguntas que este autor las realiza porque engloban todo lo que significa el buen morir para una persona. (Figueroa, 2011). Por otro lado, la discusión de la eutanasia tanto de los que se encuentra a favor como en contra se basa netamente en dos posibilidades médicas:

La legitimidad o ilegitimidad de omitir la utilización de medios extraordinarios para mantener la vida de un paciente cuya enfermedad lo lleva a la muerte. $O$ la legitimidad o ilegitimidad de auxiliar activamente a un paciente a fin de dar término anticipado a su vida, por causa de los dolores físicos o morales que invoca. (Alvear-Téllez \& Fábrega-Vega, 2006, p. 264).

Ahora bien, la eutanasia no debe exponer a las personas vulnerables a la presión de tratar de terminar con su vida para poder liberar a sus familias, los recursos médicos y no sentirse como una carga para la sociedad. Muchos de los enfermos terminales piensan que la eutanasia es la solución más idónea pero siempre y cuando sus derechos estén garantizados y no sean vulnerados. (Zúñiga-Fajuri, 2018). En Chile al igual que en muchos países, debido a casos mediáticos que se han presentado, se volvió a retomar el tema del buen morir, y de que si debía o no legalizar la misma en la constitución.

A su vez, en el mismo artículo se recalca: "En ningún caso el rechazo a tratamientos podrá tener como objetivo la aceleración artificial de la muerte, la realización de prácticas eutanásicas o el auxilio al suicidio." (Ministerio de Salud Pública, 2021, art. 16)

Tomando en cuenta el artículo 16 de Ley de Derechos y Deberes del paciente, y el Artículo 393 "El que con conocimiento de causa prestare auxilio a otro para que se suicide, sufrirá la pena de presidio menor en sus grados medio a máximo, si se efectúa la muerte". (Código Penal, 2021, Art.393).

\section{Argentina}

En el año 2007 en la provincia de Río Negro Argentina sancionó una ley en la que se encuentra contemplada el derecho de las personas a decidir: La Ley 4.264 - Muerte Digna, donde se pone énfasis a la autonomía de los pacientes para decidir sobre la 
calidad de vida que deben tener en caso de que presenten una enfermedad que pueda ocasionar mucho dolor y sufrimiento y no tenga cura, al igual esta ley indica que el paciente tiene el derecho de no aceptar cualquier método que vaya a prolongar la vida de manera artificial.

Lo más destacado de esta ley, es que se trata de eximir al médico o personal de salud de una responsabilidad civil, administrativa o penal siempre y cuando se encuentren dentro de lo que la ley establece. En el año 2008, en la provincia de Neuquén se sancionó la ley 2.611 conocida como la Ley de los Derechos del Paciente, en la cual se indicó que ...así como se respeta la vida se debe respetar la muerte, por lo que los pacientes tienen derecho a elegir de forma libre a no prolongar su vida por medio de métodos artificiales; para lo cual las entidades públicas y privadas deben contar con las instrucciones previas las cuales deben constar en documento escrito por una persona adulta, capaz y libre de tomar sus decisiones; y también tiene la libertad de revocar el consentimiento. (Luján, 2013)

En Mayo de 2012, mediante la ley 26.741 se hicieron modificaciones a la ley 26.529 , entre las cuales se puso énfasis en la autonomía del paciente para aceptar o rechazar procedimientos, medicamentos o hidrataciones que puedan alargar la misma, pero también hace indicación sobre que el paciente también puede revocar su consentimiento en cualquier momento. En el artículo 2 ibid, se hizo una definición central en cuanto a que se entiende por consentimiento informado, la declaración de voluntad, sobre

El derecho que le asiste en caso de padecer una enfermedad irreversible, incurable, o cuando se encuentre en estadio terminal, o haya sufrido lesiones que lo coloquen en igual situación, en cuanto al rechazo de procedimientos quirúrgicos, de hidratación, alimentación, de reanimación artificial o al retiro de medidas de soporte vital, cuando sean extraordinarios 0 desproporcionados en relación con las perspectivas de mejoría, o que produzcan sufrimiento desmesurado, también del derecho de rechazar procedimientos de hidratación y alimentación cuando los mismos produzcan como único efecto la prolongación en el tiempo de ese estadio terminal irreversible e incurable. (Constitución de Argentina, 2012, Art.2). 
En este sentido, (Luján, 2013), en el análisis de estos artículos, realmente prescribe un aspecto negativo que se destaca, debido al vacío jurídico con respecto a la posibilidad de los médicos para que les sea respetado igualmente su derecho a la objeción de conciencia, en los eventos en que no se encuentren de acuerdo con los pacientes y/o sus familiares, ante el rechazo y/o retiro de soporte vital e hidratación. (p. 35).

\section{Colombia}

En este ámbito territorial se tiene que "La eutanasia es un tema de gran discusión en Colombia, pero no es fácil puntualizar sobre ella. El desconocimiento general que se tiene y el hecho de tratar directamente con la muerte, presenta uno de sus más graves problemas." (Delgado-Rojas, 2016, p. 227). En el caso colombiano, la eutanasia como práctica encaminada a garantizar la muerte en condiciones dignas, fue despenalizada mediante la sentencia 239 de 1997, pero bajo un modelo regulativo en cuanto a la condición del sujeto pasivo, la modalidad de la solicitud y la condición del sujeto activo.

En tal sentido, se estableció que debía existir una constatación rigurosa de la condición real del paciente por parte de personas competentes, en cuanto a lo terminal de su patología, el carácter unívoco de su decisión expresada a través de una solicitud clara y libre contenida además en su consentimiento otorgado y el estatus de médico que debe ostentar quien está llamado a practicarla (Corte Constitucional, C-239 de 1997).

Lo anterior quiere decir, que el Estado colombiano tiene un avance y un desarrollo considerablemente admirable en términos del respeto por la muerte digna a través de la eutanasia, la cual es perfectamente viable en la actualidad, para personas en una situación de enfermedad terminal como para personas con afectaciones sico-físicas incurables generadoras de graves e intensos sufrimientos.

Se puede inferir hasta este punto que, en lo concerniente a Chile y Argentina, aunque la eutanasia no esté legalizada de forma expresa, sí cuentan regulaciones en cuanto a la asistencia frente al fenómeno de la muerte en condiciones de dignidad, en el marco de las regulaciones jurídicas internas, y sobre todo, se trata de un asunto que se encuentra 
en fuertes debates que al menos generan una esperanza de que, en cualquier momento, pueda abrirse la puerta al respeto de la autonomía de quien padece una enfermedad que le resta dignidad a su vida.

En el caso colombiano, la situación está mucho más avanzada, pues tal práctica se aplica tanto a las enfermedades terminales como a aquellas que no lo son -tanto a nivel físico como síquico-, siempre y cuando atenten contra el precepto de una vida digna. E Ecuador, a diferencia de los países analizados no cuenta con una legislación ni con propuestas para el derecho a una muerte digna a través de la eutanasia, lo cual hace necesario que el debate se plantee con urgencia, para dar un nuevo paso evolutivo en el sistema jurídico a nivel interno.

\section{La bioética y su influencia en la eutanasia}

La ética médica se basa en 4 fundamentos o principios muy importantes como son la no maleficencia, la beneficencia, la autonomía y la Justicia. Siguiendo a Gómez, debe indicarse que por no maleficencia se hace referencia al respeto a la integridad de una persona, evitar el riesgo innecesario y los riesgos excesivos, es decir hace referencia al principio básico de primun non nocere permitiendo que el médico se oponga algún procedimiento que él crea innecesario a pesar de que la persona lo hay solicitado, pero al mismo tiempo tiene la obligación de referir a otro colega quien tenga la capacidad para realizar. La beneficencia implica la obligación de hacer el bien al paciente, velar por su salud, como lo indica en el juramento hipocrático. Por la justicia, debe entenderse que se basa en el reparto equitativo de beneficios, actuar con equidad, para así evitar la discriminación en los recursos de salud, bajo la idea de que todos los pacientes tienen los mismos derechos y los mismos deberes.

Finalmente, la autonomía es uno de los principios más relevantes, porque implica la obligación de respetar las opiniones y decisiones que tome cada persona en lo que atañe a su vida, tratamiento médico, o procedimientos que se realicen. Este principio en cual se constituye básicamente el consentimiento informado en el cual el paciente toma la 
libre decisión sobre su propio bien, y que no sea impuesto en contra de su voluntad ya sea por la fuerza o por aprovecharse de la ignorancia de las personas. (Gómez-Sánchez, 2009).

En una declaración publicada en 1993 por el Foro Mundial de la Salud, basada en la experiencia en Bolonia sobre personas con cáncer avanzado, se reportó que unas veinticinco mil personas le solicitaron a sus médicos garantías para morir en un determinado momento su tratamiento les resultase insufrible. (Baum, 2017, p.16). En relación a esta declaración, la empatía no sólo por parte del personal médico sino también de los que manejan la legislación debería hacer que hagan una mirada hacia las personas con estos sufrimientos debido a las enfermedades que la adolecen. Los principios pueden ser utilizados generalmente, pero en casos concretos como el derecho a la eutanasia podrían ser una ayuda para determinar si es importante o no este derecho.

Los cuatro principios explicados hacen referencia a diferentes aspectos que deben ser articulados de la mejor manera. Por ejemplo, el principio de autonomía se basa netamente al paciente, el principio de beneficencia y no maleficencia se basa en lo que el médico debe realizar, es decir el acto médico en sí, hacer lo que necesita el paciente sin más ni menos; el principio de justicia se refiere a las obligaciones que el estado debe tener para con los pacientes que acuden a los servicios de salud, se debe encargar de proporcionar todos los medios y presupuestos para una atención adecuada y eficaz.

En el concepto de principio de no maleficencia, se indica claramente que se debe respetar la integridad de un paciente -no realizar daño en él-, pero también hace relación a un riesgo innecesario y los riesgos excesivos, como podría ser el que se le cause a un paciente con una enfermedad catastrófica, terminal, huérfana que provoque daño físico o psicológico cual sería el riesgo innecesario o un riesgo excesivo, a quien se le proporcionen tratamientos largos que provocan dolores o síntomas que las personas no pueden tolerar, entonces para cada persona el riesgo innecesario o excesivo tiende a ser un concepto con variedad de ideas. 
El principio de beneficencia se basa el juramento hipocrático, pero en el hacer el bien al paciente también se debe tener en cuenta cuál es el bien de cada persona, si un médico realiza un procedimiento o inicia un tratamiento por el bien del paciente y este lo cumple y llega a su curación se ha cumplido adecuadamente, pero qué sucede con los pacientes en fases terminales de sus enfermedades -físicas o psicológicas-; ¿hasta dónde llega el bien que se puede realizar mediante un procedimiento?

Si el paciente decide continuar con su tratamiento, pues se debe hacer lo que el paciente decide porque es su derecho a elegir; pero si el paciente no desea continuar con tratamiento que para él sólo le ocasiona dolor, tristeza, sufrimiento también debe respetarse su derecho a elegir. Por esto, el principio de beneficencia debe ser claramente articulado con el principio de autonomía, es decir el derecho a decidir si aceptar o proseguir con los tratamientos indicados. Ahora también hay que aclarar que el sentido de respetar la autonomía de las personas no quiere decir, que el derecho a la eutanasia no puede ser considerado como un derecho al homicidio, sino una respuesta al sufrimiento extremo de una persona que se encuentran en el final de su vida.

\section{CONCLUSIONES}

En el Ecuador no existe legislación vigente en cuanto a lo que se refiere a la eutanasia, a pesar de que existen enfermedades catastróficas, raras o huérfanas, terminales que no son de estudiadas a nivel de esta práctica, enfermedades las cuales han sido abandonadas y que los personas que los padecen pueden presentar una vida ajena a las condiciones de dignidad que merece el ser humano.

$\mathrm{Si}$ el bien mayor en el Ecuador es una vida digna, las personas con enfermedades que tengan alteraciones graves a nivel de su integridad física y psicológica deben tener garantizado este derecho desde una dimensión real, y no simplemente desde la simpleza de la existencia biológica.

Los principios de bioética ayudan a formar médicos al servicio de sus pacientes, pero cuando se trata de la eutanasia, se aprecia una superficialidad de su aplicación en la 
garantía y el respeto por la dignidad de las personas quienes solicitan legítimamente su derecho a la eutanasia.

A pesar de la experiencia cercana de algunos países suramericanos como Chile, Argentina y Colombia, en el Ecuador hace falta re abrir el debate sobre la eutanasia como derecho fundamental, pues sin interesar que se esté a favor o en contra, siempre será importante el diálogo y la dialéctica como forma razonable para llegar a consensos.

\section{FINANCIAMIENTO}

No monetario.

\section{AGRADECIMIENTO}

A la Universidad Católica de Cuenca; por motivar el desarrollo de la investigación.

\section{REFERENCIAS CONSULTADAS}

Acuerdo Ministerial 1829. Inclusión de enfermedades consideradas catastróficas, raras y huérfanas para beneficiarios del bono Joaquín Gallegos Lara [Inclusion of diseases considered catastrophic, rare and orphan for beneficiaries of the Joaquín Gallegos Lara voucher]. Registro Oficial 798 de 27-sep-2012 Ultima modificación: 18-abr2013. Recuperado de https://n9.cl/juw8t

Alvear-Téllez J. \& Fábrega-Vega, H. (2006). Análisis crítico del proyecto de ley sobre eutanasia y sus antecedentes legislativos. Revista Actualidad Jurídica $N^{\circ} 14$. https://n9.cl/90und

Baños, R. (2014). La eutanasia y su legalización como una opción en la legislación ecuatoriana [Euthanasia and its legalization as an option in Ecuadorian legislation]. http://www.dspace.uce.edu.ec/handle/25000/3210

Barahona-Quesada, M. (2013). El papel de la investigación teórica en la construcción del conocimiento [The role of theoretical research in the construction of knowledge.]. Revista Rupturas, 3(1), 2-16. https://doi.org/10.22458/rr.v3i1.254

Baum, E. (2017). Eutanasia, empatía, compasión y Derechos Humanos [Euthanasia, empathy, compassion and Human Rights]. Revista de Bioética y Derecho, (39), 521. 
Bou-Torrent, R. (2020). Osteogénesis imperfecta [Imperfect osteogenesis]. Protoc diagn ter pediatr, 2, 349-359. https://n9.cl/3bi2x

Bucheli, M. (2012). Esclerosis Lateral Amiotrófica: Criterios de El Escorial y la Electromiografía en su Temprano Diagnóstico [Amyotrophic Lateral Sclerosis: Criteria of El Escorial and Electromyography in its Early Diagnosis]. Revista Ecuatoriana de Neurología, 21, 1-3. https://n9.cl/a7pku

Bustíos, C. (2007). Encefalopatía hepática [Hepatic encephalopathy ]. Acta Médica Peruana, 24(1), 40-46.

Chávez RE, \& Castillo MRC. (2013). Revisión bibliográfica sobre VIH/sida [Literature review on HIV / AIDS]. Mul Med, 17(4):1-25. https://n9.cl/jse03

Código de Ética Médica. Acuerdo Ministerial 14660 Registro Oficial 5 de 17-ago.-1992. Recuperado de https://n9.cl/73snd

Código Penal 2021. Art.393. recuperado de https://n9.cl/g6sc

Constitución de Argentina. (1994). Boletín Oficial, 23 de agosto de 1994. Recuperado de https://n9.cl/h4tijp

Constitución de la República de Ecuador (2008). Articulo 66. Recuperado de https://n9.cl/ahhel

Corte Constitucional, C-239 de 1997. Homicidio por piedad-elementos/homicidio pietistico o eutanasico/homicidio eugenésico [Pity-element homicide / pietistic or euthanasic homicide / eugenic homicide]. Recuperado de https://n9.cl/7xbom

Creagh, M. (2012) Dilema ético de la eutanasia [Ethical dilemma of euthanasia]. Revista Cubana de Salud Pública, 38(1), 150-155.

Delgado-Rojas, E. (2017). Eutanasia en Colombia: una mirada hacia la nueva legislación [Euthanasia in Colombia: a look at the new legislation]. Justicia, 22(31), 226-239. http://dx.doi.org/10.17081/just.22.31.2608

Domínguez MR, Morales EM, Rossiere ENL, et al. Esclerosis múltiple: revisión de la literatura médica [Multiple sclerosis: a review of the medical literature]. Rev Fac Med UNAM, 55(5):26-35. https://n9.cl/vziwg 
Dulzaides-Iglesias, M, \& Molina-Gómez, A. (2004). Análisis documental y de información: dos componentes de un mismo proceso [Documentary and information analysis: two components of the same process]. ACIMED, 12(2), 1.

Esquivel, J. (2003). El derecho a una muerte digna: la eutanasia [The right to a dignified death: euthanasia]. Recuperado de https://n9.cl/1ykg

Figueroa, R. (2011). Matar, dejar morir y eutanasia en el proyecto de ley sobre derechos de las personas y en la doctrina chilena [Killing, letting die and euthanasia in the bill on people's rights and in Chilean doctrine]. Revista médica de Chile, 139(5), 655659. https://dx.doi.org/10.4067/S0034-98872011000500014

Figueroa, F. (2016). Síndrome de Cotard [Cotard's Syndrome]. https://n9.cl/tbgrm

Flier, D. (2021). Eutanasia legal: la Argentina empieza a debatir el derecho a morir

Formiga, F., Robles, M. J., \& Fort, I. (2009). Demencia, una enfermedad evolutiva: demencia severa. Identificación de demencia terminal [Dementia, a progressive disease: severe dementia. Identification of end-stage dementia]. Revista espanola $\begin{array}{lllll}\text { de } & \text { geriatria } y & \text { gerontologia, } 44 & \text { Suppl }\end{array}$ https://doi.org/10.1016/j.regg.2009.05.009.

Gómez-Sánchez, P. (2009). Principios básicos de bioética [Basic principles of bioethics]. Revista Peruana de Ginecología y Obstetricia, 55(4),1-12. .

González-Rodríguez, M. (2013). Espondilitis anquilosante: conceptos generales [Ankylosing spondylitis: general concepts]. Residente, ;8(3):106-113. https://n9.cl/yeh40

Guairacaja, A. (2011). Proyecto de legislación de la eutanasia, por la protección al derecho a la vida digna, en la legislación ecuatoriana [Draft legislation on euthanasia, for the protection of the right to a dignified life, in Ecuadorian legislation]. Trabajo de graduación [Tesis de pregrado. Universidad técnica de Cotopaxi]. http://repositorio.utc.edu.ec/handle/27000/353

Luján, V (2013). El Derecho a una muerte digna en la legislación argentina [The Right to a dignified death in Argentine law]. Recuperado de https://n9.cl/11 gig

Martín, J. (2009). Guía Práctica clínica para el tratamiento de la Esquizofrenia en centros de salud mental [Clinical Practice Guide for the treatment of Schizophrenia in mental health centers]. Servicio murciano de salud. https://n9.cl/cdi9e 
Matias-Camargo, S. R. (2012). La investigación jurídica, su necesidad y su impacto [Legal research, its need and its impact]. Diálogos De Saberes, (37), 11-22.

Médica.

Mendoza, M. (2018). Manifestaciones respiratorias en la fibrosis quística [Respiratory manifestations in cystic fibrosis]. Adolescere, VI(3): 52.e1-52.e8. https://n9.cl/k5muc

Mera, S. (2017) El derecho a morir dignamente dentro de la legislación ecuatoriana [Tesis de pregrado. Pontificia Universidad Católica del Ecuador Sede Amba]

Millás-Mur, J. (2019). Ética y Bioética en el pregrado de Medicina: una propuesta [Ethics and Bioethics in Medicine undergraduate: a proposal]. Revista Peruana de Medicina $\begin{array}{lllll}\text { Experimental } y & \text { Salud } & \text { 9ública, }\end{array}$ https://doi.org/10.17843/rpmesp.2019.361.4260

Ministerio de Salud Chile. (2021). Regula los Derechos y Deberes que tienen las personas en relación con acciones vinculadas a su atención en salud [Regulates the Rights and Duties that people have in relation to actions related to their health care]. Recuperado de https://n9.cl/aki7de

Munné-Díaz, C. (2013). Enfermedad de Creutzfeldt-Jacob [Creutzfeldt-Jakob disease]. Revista Científica de la Sociedad Española de Enfermería Neurológica, 37(1), 2932. https://doi.org/10.1016/j.sedene.2013.04.006

Muñoz-Collazos, M. (2010). Enfermedades cerebrovasculares (ECV) [Cerebrovascular disease]. Acta Neurológica Colombiana, 26(2), 59-61.

Navarro-Sanz, J. (s.f). Cuidados paliativos no oncológicos: enfermedad terminal y factores pronósticos [Non-cancer palliative care: terminal illness and prognostic factors]. Sociedad Española de Cuidados Paliativos. Recuperado de https://n9.cl/738sh

Resolución 971 de 2021. Recuperado de Resolución 971

Rodríguez, J., Díaz, Y., Rojas, Y., Rodríguez, Y. \& Núñez, E. (2013). Actualización de Enfermedad de Huntington [Update on Huntington Disease]. Correo Científico Médico, 17(Supl. 1), 546-557.

Varela-Jiménez, R., Jiménez, A., Carrillo, D., Carrillo, E. \& Rodríguez, S. (2015). Síndrome Hepatorrenal [Hepatorenal syndrome]. Revista Médica MD, 6 (2), 135140. https://n9.cl/gh382 
Vives, R., Sorlí, J., Sierra, L. \& García, M. (2015). Angioedema Hereditario [Hereditary angioedema]. Revista Clínica de Medicina de Familia, 8(1), 6265. https://dx.doi.org/10.4321/S1699-695X2015000100008

Zúñiga-Fajuri, A. (2008). Derechos del Paciente y Eutanasia en Chile [Patient's rights and euthanasia in Chile]. Revista de derecho (Valdivia), 21(2), 111-130. https://dx.doi.org/10.4067/S0718-09502008000200005

Zúñiga-Fajuri, A. (2018). Eutanasia y justicia distributiva sanitaria: aportes a la discusión moral sobre el derecho a la buena muerte [Euthanasia and health distributive justice: contributions to the moral discussion on the right to a good death]. Revista de Ciencias Sociales, 0(73). https://doi.org/10.22370/rcs.2018.73.2266

C2021 por los autores. Este artículo es de acceso abierto y distribuido según los términos y condiciones de la licencia Creative Commons Atribución-NoComercial-Compartirlgual 4.0 Internacional (CC BY-NC-SA 4.0) (https://creativecommons.org/licenses/by-nc-sa/4.0/). 